\title{
Sulindac attenuates valproic acid-induced oxidative stress levels in primary cultured cortical neurons and ameliorates repetitive/stereotypic-like movement disorders in Wistar rats prenatally exposed to valproic acid
}

\author{
YINGHUA ZHANG ${ }^{1,2}$, CAILING YANG ${ }^{3}$, GUOYAN YUAN $^{3}$, \\ ZHONGPING WANG ${ }^{2,4}$, WEIGANG CUI ${ }^{1,2}$ and RUIXI LI $^{2}$

\begin{abstract}
${ }^{1}$ Department of Human Anatomy, Xinxiang Medical University, Xinxiang, Henan 453003; ${ }^{2}$ Department of Anatomy, Xinxiang Medical University, Weihui, Henan 453100; ${ }^{4}$ Department of Physiology and Pathophysiology,

School of Basic Medical Science, Jiujiang University, Jiujiang, Jiangxi 332000, P.R. China
\end{abstract} \\ Histology and Embryology, Shanghai Medical College, Fudan University, Shanghai 200032; ${ }^{3}$ The First Affiliated Hospital,
}

Received July 6, 2014; Accepted October 31, 2014

DOI: $10.3892 /$ ijmm.2014.1996

\begin{abstract}
Accumulating evidence suggests that anti-inflammatory agents and antioxidants have neuroprotective properties and may be beneficial in the treatment of neurodevelopental disorders, such as autism. In the present study, the possible neuroprotective properties of sulindac, a non-steroidal antiinflammatory drug (NSAID), were investigated in vitro using cultured cortical neurons with valproic acid (VPA)-induced neurotoxicity, as well as in vivo through the behavioral analysis of rats prenatally exposed to VPA as a model of autism. VPA induced 4-hydroxynonenal (4-HNE) expression, reactive oxygen species (ROS) generation and decreased cell viability in primary cultured cortical neurons established from timed-pregnant (embryonic day 18) Wistar rat pups. However, co-incubation of the neurons with VPA and sulindac reduced oxidative stress and increased cell viability. The rats were administered an intraperitoneal injection with one of the following: VPA, sulindac, VPA and sulindac, or physiological saline, and their offspring were subjected to the open field test. During the test trials, repetitive/stereotypic-like movements for each rat were recorded and analyzed. The results revealed that treatment with both sulindac and VPA reduced the VPA-induced repetitive/stereotypic-like activity and the sulindac and VPA-treated animals responded better in the open field test compared to the VPA-treated animals. The results from the present study demonstrate that the antioxidant properties of sulindac may prove to be beneficial in the treatment of
\end{abstract}

Correspondence to: Dr Yinghua Zhang, Department of Human Anatomy, Xinxiang Medical University, 601 Jinsui Road, Xinxiang, Henan 453003, P.R. China

E-mail: zyhflo2013@163.com

Key words: oxidative stress, valproic acid, Wnt/ $\beta$-catenin pathway, autism, sulindac autism, suggesting that the upregulation of the Wnt/ $\beta$-catenin signaling pathway disrupts oxidative homeostasis and facilitates susceptibility to autism.

\section{Introduction}

Three defining clinical symptoms of autism are aberrant reciprocal social interactions, deficits in social communication and repetitive/stereotypic behavior $(1,2)$. The etiology of autism remains largely unclear thus far. With the continuous accumulation of research on the molecular pathogenesis of autism, more attention is being paid to the canonical Wnt signaling pathway $(3,4)$. The canonical Wnt pathway has been implicated in a variety of cellular functions, such as cell proliferation, differentiation, apoptosis and neuronal migration during embryonic development (5). Given this broad spectrum of roles, it is no surprise that the dysregulation of the canonical Wnt signaling pathway may cause a similarly broad spectrum of deleterious effects on neuronal development, and largely on this basis may thereby contribute to the pathogenesis of neurodevelopmental disorders, such as autism.

A number of previous studies have suggested that the canonical Wnt pathway is involved in autism and the dysfunction of the Wnt signaling pathway induces morphological and functional abnormalities similar to those observed in autistic individuals. For example, transgenic mice expressing active stabilized forms of $\beta$-catenin in neuronal precursor cells have been shown to develop grossly enlarged brains with an increased cerebral cortical volume (6), whereas the inactivation of the $\beta$-catenin gene causes marked brain malformation and the failure of craniofacial development (7). The upregulation of $\beta$-catenin expression, as well as that of its downstream target genes $(3)$, has been observed in rat models of autism $(3,4)$, and the expression levels of $\beta$-catenin are abnormal with changes over time and space. The mutation of genes, such as Wnt1, that modestly increase the activation of this downstream signaling pathway would therefore be expected to result in neurodevelop- 
mental and behavioral phenotypes. A recent study suggested that a Wnt1 missense variant leading to the Wnt signaling pathway activation contributes to autism in the context of other genetic and non-genetic factors that increase susceptibility to autism (8).

Recent studies have focused, in particular, on the role of oxidative stress in autism. In fact, oxidative stress has also been implicated in the pathogenesis of other neuropsychiatric diseases, including schizophrenia $(9,10)$, a disease that may be the genetic opposite of autism (11). Accordingly, accumulating evidence suggests a role of oxidative stress in the development and clinical manifestation of autism $(1,2)$.

Sulindac is an FDA-approved non-steroidal anti-inflammatory drug (NSAID) with documented anticancer activities. Sulindac, as a NSAID with a number of functions, has been used as a specific Wnt pathway inhibitor and is known as a pharmacological inhibitor of $\beta$-catenin for the prevention of metastasis $(12,13)$. Since the upregulation of the canonical Wnt pathway has been associated with the pathogenisis of autism and valproic acid (VPA) is known to generate autism phenotypes, in this study, we therefore used VPA to generate conditions similar to those observed in autism. We aimed to determine whether sulindac ameliorates autism-like behavioral abnormalities, such as repetitive/stereotypic-like movement deficits that develop in rats prenatally exposed to valproic acid (VPA) as an animal model of autism. In addition, we aimed to identify the possible mechanisms responsible for the development of autism using primary cortical neurons.

\section{Materials and methods}

Animals and exposure to VPA. All animal experiments were carried out in accordance with the National Institutes of Health guidelines for the Care and Use of Laboratory Animals and the regulations of Fudan University (Shanghai, China) for animal experimentation. The experimental protocol was approved by the Shanghai Medical Experimental Animal Care and Use Committee. As described in our previous study (4), with some modifications, Female Wistar rats (Shanghai Institute of Materia Medica, Chinese Academy of Sciences, Shanghai, China) were mated overnight, and the morning on which spermatozoa were found was designated as the first day of gestation. The female fats received a single intraperitoneal injection of $600 \mathrm{mg} / \mathrm{kg}$ VPA (CAS no: 1069665, Sigma, St. Louis, MO, USA) or $5 \mathrm{mg} / \mathrm{kg}$ sulindac (CAS:38194502, Sigma) following the administration of $600 \mathrm{mg} / \mathrm{kg}$ VPA for $30 \mathrm{~min}$ on day 12.5 after conception, and the control female rats were injected with physiological saline or $5 \mathrm{mg} / \mathrm{kg}$ sulindac at the same time point. The offspring were weaned on postnatal day (PND)23, and rats of either gender were housed separately. Repetitive/stereotypiclike behavioral tests were conducted for all animals during the adolescent period. The behavioral experiments were performed in the light phase between 09:00 and 15:00.

Primary neuronal culture and experimental treatments. Wistar rats were used in the present study. The experiments were carried out in accordance with the regulations of Fudan University for animal experimentation. Cortices were dissected from timed-pregnant (embryonic day 18) Wistar rat pups, dissociated in a solution of $2.5 \mathrm{mg} / \mathrm{ml}$ trypsin (Gibco, Carlsbad, CA, USA) for $15 \mathrm{~min}$, and resuspended following centrifugation for 5 min in DMEM/F12 (Gibco) supplemented with $10 \%$ fetal bovine serum, $1 \%$ penicillin/streptomycin and $0.5 \mathrm{mmol} / 1$ glutamine. The cells were plated on 6 -well plates pre-treated with poly-L-lysine $(100 \mu \mathrm{g} / \mathrm{ml}, \mathrm{CAS}$ no: 25988630 , Sigma). Twenty-four hours later, the culture medium was completely replaced with NB/B27 (neurobasal medium supplemented with $2 \%$ B27, $1 \%$ penicillin/streptomycin and $0.5 \mathrm{mmol} / \mathrm{l}$ glutamine). Subsequently, approximately one-third of the volume was removed and replaced with an equal volume of fresh NB (0050128DJ, Gibco)/B27 (10889-038, Gibco) twice per week. On the 7th day of plating, the primary cultured neurons were exposed to VPA. After $24 \mathrm{~h}$, VPA was replaced with the antioxidant, N-acetylcysteine (NAC; CAS no: 616911, Sigma), or sulindac for $1 \mathrm{~h}$. The neurons were then harvested for further assay.

$R N A$ extraction and reverse transcription-quantitative PCR $(R T-q P C R)$. RT-qPCR. RT-qPCR was carried out as described in our previous study with minor modifications (4). Total RNA was isolated from the cultured cortical neurons using TRIzol reagent (15596-018, Gibco). Subsequently, cDNA was prepared using the PrimeScript ${ }^{\mathrm{TM}}$ RT reagent kit (DRR019A, Takara Biotechnology, Dalian, China). The primer sequences for RT-qPCR are listed in Table I. PCR parameters were as follows: 29 cycles of denaturation at $95^{\circ} \mathrm{C}$ for $30 \mathrm{sec}$, annealing at $57^{\circ} \mathrm{C}$ for $30 \mathrm{sec}$ and extension at $72^{\circ} \mathrm{C}$ for $30 \mathrm{sec}$. The realtime detection of the emission intensity of SYBR-Green I bound to double-stranded DNA was performed using the Rotor-Gene 3000 real-time DNA analysis system (RG-3000, Corbett Research, Sydney, Australia). At the end of the runs, melting curves were obtained to make sure there were no primer-dimer artifacts.

The PCR assay was performed simultaneously with standard samples to construct each standard curve. Referring to the standard curve, the relative mRNA expression level was normalized to the expression level of the endogenous reference, GAPDH, to calibrate the possible variability in the initial amount of total RNA in each sample.

Western blot analysis. Western blot analysis was performed as previously described (4), with some modifications. Briefly, equal amounts of protein (25-30 $\mu \mathrm{g})$ from cell lysates were separated by sodium dodecylsufate (SDS)-polyacrylamide gel electrophoresis (PAGE) and transferred onto PVDF membranes (Millipore Corp., Bedford, MA, USA). The blots were incubated with the primary antibodies in 5\% BSA or fat-free milk in TBST buffer overnight at $4^{\circ} \mathrm{C}$. Bound primary antibodies were visualized using horseradish peroxidaselabeled secondary antibodies and were detected by enhanced chemiluminescence (ECL). The densities of the protein bands were quantified using ImageQuant software (LAS-4000, GE Healthcare, Fairfield, CT, USA). GAPDH (1:5000, Kangen Biotechnology Co., Shanghai, China) was used to confirm equal loading. The primary antibodies used were as follows: antiglycogen synthase kinase (GSK)-3 $\beta$ (1:1000, Cat no. 9315; Cell Signaling Technology, Danvers, MA, USA), phospho-GSK-3 $\beta$ (Ser9) (1:500, Cat no. 9336; Cell Signaling Technology), anti- $\beta$-catenin (1:1000, sc-7199; Santa Cruz Biotechnology, San Diego, CA, USA), phospho- $\beta$-catenin (Ser33/37/Thr41) (1:1000, Cat no. 9561; Cell Signaling Technology) and anti- 
Table I. Primer sets used in RT-qPCR.

\begin{tabular}{lll}
\hline Primer name & Accession no. & \multicolumn{1}{c}{ Sequence 5' $\rightarrow 3^{\prime}$} \\
\hline GSK-3 $\beta$ & NM_032080.1 & $\begin{array}{l}\text { Forward: GTTGGTGGAAATAATAAAGG } \\
\text { Reverse: AAGTTGAAGAGGGCAGGT }\end{array}$ \\
$\beta$-catenin & NM_053357.2 & $\begin{array}{l}\text { Forward: CTGAGAAACTTGTCCGATGC } \\
\text { Reverse: CGGTAATGTCCTCCCTGT }\end{array}$ \\
GAPDH & NM_017008.3 & Forward: TATCGGACGCCTGGTTAC \\
& & Reverse: TGCTGACAATCTTGAGGGA \\
\hline
\end{tabular}

4-hydroxynonenal (4-HNE) (1:500, MAB3249; R\&D Systems, Minneapolis, MN, USA).

Cell viability assay. Cell survival was evaluated by MTT assay as previously described (14). Briefly, the cells were plated into 96-well microtiter plates and exposed to VPA or sulindac for $48 \mathrm{~h}$. Following exposure to VPA or sulindac, $10 \mu \mathrm{l}$ of MTT labeling reagents were added to each well and the plates were incubated at $37^{\circ} \mathrm{C}$ for $4 \mathrm{~h}$. The cultures were then solubilized, and the spectrophotometric absorbance of the samples was detected using a microtiter plate reader. The absorbance was read at $570 \mathrm{~nm}$.

Measurement of intracellular ROS levels. The average level of intracellular ROS was evaluated in the cells loaded with the redox-sensitive dye, 2'-7'-dichlorodihydrofluorescein diacetate (DCFH-DA; Molecular Probes, Eugene, OR, USA). An increase in the fluorescence emission of dichlorofluorescein (DCF), a derivative of DCFH-DA, reflects the enhanced cellular oxidative stress. All the experimental cells were washed 3 times with PBS for $5 \mathrm{~min}$, stained in the dark for $20 \mathrm{~min}$ with $10 \mu \mathrm{mol} / \mathrm{l}$ DCFH-DA and harvested. Fluorescence was observed under fluorescence light microscope, and measured at an excitation and emission wavelength of 485 and $530 \mathrm{~nm}$, respectively using a fluorescence activated cell sorting (FACS) on fluorescence spectrometer (HTS 7000, Perkin Elmer, Boston, MA, USA).

Behavioral testing: open field test. Exploratory activity was assessed in an automated open field apparatus (RS Medical Associates, PA, USA) for rats. All rats were tested one by one in an open field apparatus with an area of $100 \times 100 \mathrm{~cm}^{2}$ and a height of $40 \mathrm{~cm}$. Testing was conducted under dim light (approximately 80 lux) during the morning hours in an undisturbed room and no stressor was applied to the animals for at least $12 \mathrm{~h}$ before the test. Two rats were tested at one time, one animal per chamber. A rat was placed in the center of the apparatus and then allowed to behave and move freely for $30 \mathrm{~min}$. At the same time, movements were recorded using a digital video camera and recorded for further analysis. Data were assessed by comparing the changes in the mean activity across a 30-min testing session divided into 6 time bins $(5 \mathrm{~min}$ each). Grooming and rearing behaviors were also scored in the same paradigm by an observer blinded to the treatments. The time spent self-grooming and the number of rearing episodes were scored, as previously described (15). A rearing episode is defined as the number of times the rat stands upright on its hind limbs. Repetitive/stereotypic-like behaviors were scored and quantified by an experimenter blinded to the treatments.

Statistical analysis. All values are presented as the means \pm SEM. Multiple group comparisons were performed by one-way analysis of variance (ANOVA) with post-hoc correction. Repeated measures between 2 factors were examined using a repeated two-way analysis of variance (ANOVA) and Fisher's least-significant difference post hoc comparison using SPSS 16.0 software (SPSS, Inc., Chicago, IL, USA). Values of $\mathrm{p}<0.05$ were considered to indicate statistically significant differences.

\section{Results}

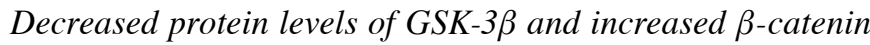
expression. Whether VPA activates the canonical Wnt pathway remains unknown. To determine the effects of VPA on the activity of the Wnt/ $\beta$-catenin signaling pathway, dissociated primary cortical neurons were cultured. Primary cultured cortical neurons were exposed to VPA $(1,5$ and $10 \mathrm{mmol} / \mathrm{l})$ for $24 \mathrm{~h}$. The cortical neurons were continuously exposed to growth medium as the control. Subsequently, all the abovementioned cultured cortical neurons were processed for protein extraction. Quantitative analysis of the protein content by western blotting demonstrated that VPA induced a concentration-dependent decrease in the expression of GSK-3 $\beta$, but an increase in the expression of $\beta$-catenin when compared to the controls (Fig. 1A and B). As shown in Fig. 1A, $5 \mathrm{mmol} / 1$ and $10 \mathrm{mmol} / \mathrm{l} \mathrm{VPA}$ increased the protein levels of $\beta$-catenin compared with the controls. We then investigated GSK-3 $\beta$, a negative regulatory factor of $\beta$-catenin. The results revealed that GSK-3 $\beta$ expression was lower in the 1,5 and $10 \mathrm{mmol} / 1 \mathrm{VPA}$-treated groups in comparison to the controls; however, $5 \mathrm{mmol} / \mathrm{l}$ and $10 \mathrm{mmol} / \mathrm{l}$ VPA significantly decreased the protein levels of GSK-3 $\beta$ (P<0.001; Fig. 1B). Therefore, we selected the dose of $5 \mathrm{mmol} / \mathrm{l}$ VPA as an ideal concentration for further experiments.

Effects of NAC on VPA-treated primary cultured neurons. Primary cultured neurons were exposed to VPA $(5 \mathrm{mmol} / \mathrm{l})$. After $24 \mathrm{~h}$, VPA was replaced with the antioxidant, NAC $(1 \mathrm{mM})$, for $1 \mathrm{~h}$. The neurons were then harvested for further assay. Western blot analysis, RT-qPCR and flow cytometry (FACS analysis) were used to further investigate the levels of 4-HNE, GSK-3 $\beta$, $\beta$-catenin and ROS. The results from western blot analysis and FACS revealed that treatment with VPA increased the levels of 4-HNE and ROS as compared with the controls (Fig. 2A and B). 
A
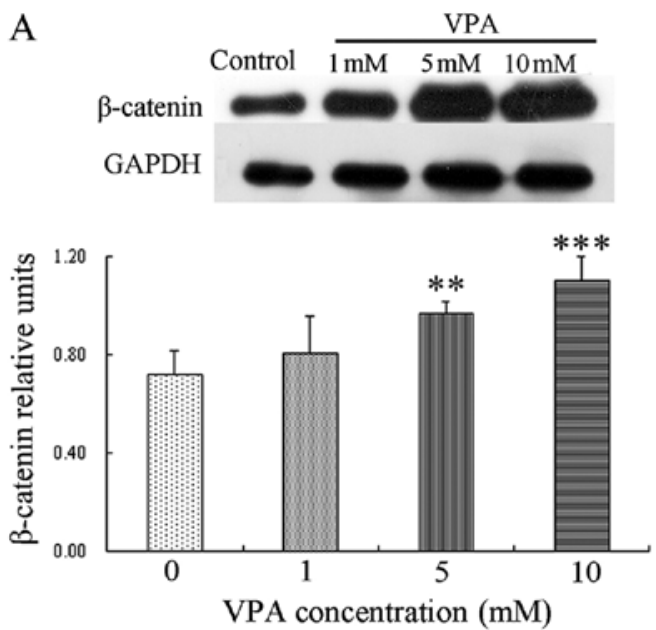

B
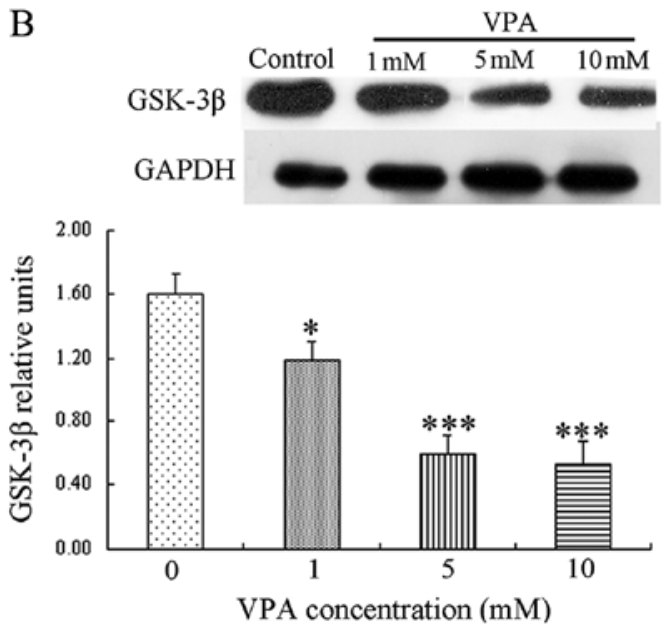

Figure 1. Valproic acid (VPA) increases $\beta$-catenin, but decreases GSK-3 $\beta$ levels in primary cultured neurons. Data are expressed as the means \pm SEM, ${ }^{*} \mathrm{P}<0.05$, ${ }^{* *} \mathrm{P}<0.01,{ }^{* * *} \mathrm{P}<0.001$ vs. control group.

A

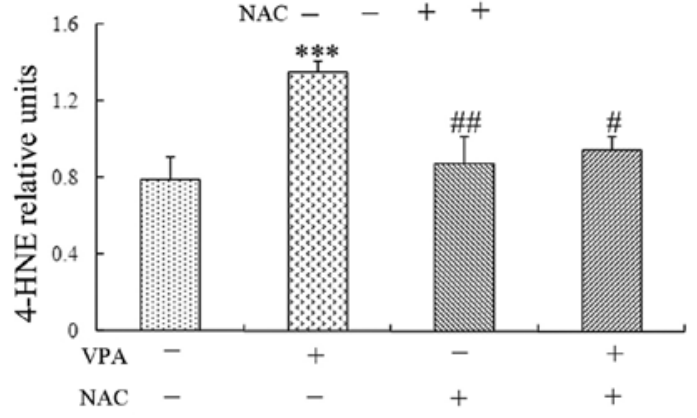

B

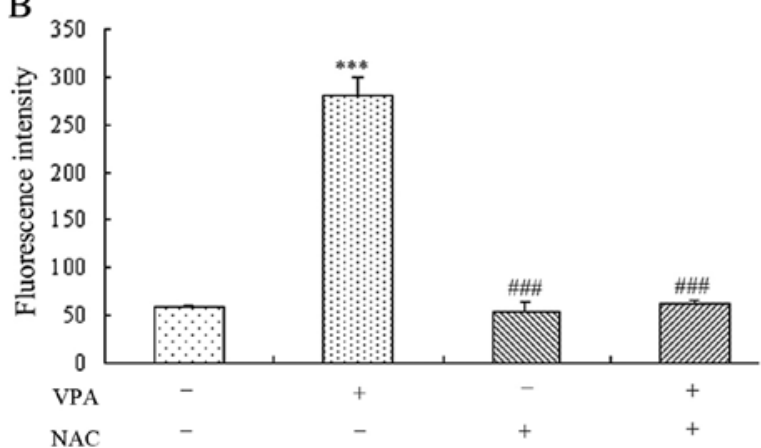

$\mathrm{C}$
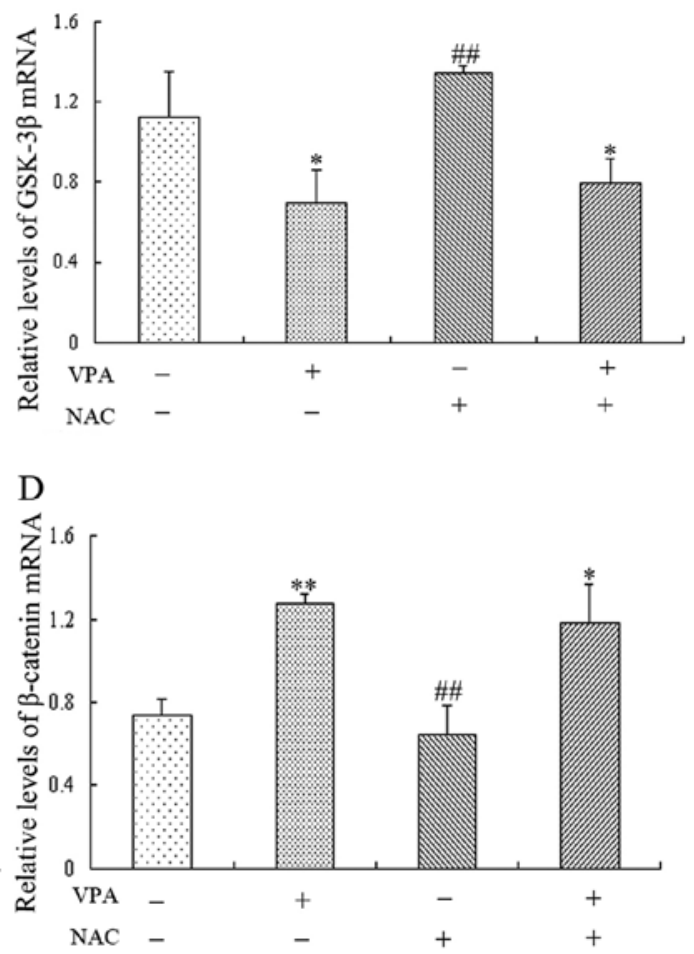

Figure 2. Effects of N-acetylcysteine (NAC) on valproic acid (VPA)-treated neurons. (A) Western blot analysis of 4-hydroxynonenal (4-HNE) expression. (B) Representative fluorescence intensity for reactive oxygen species (ROS) by fluorescence activated cell sorting (FACS). (C and D) RT-qPCR analysis of glycogen synthase kinase (GSK)-3 $\beta$ and $\beta$-catenin expression. ${ }^{*} \mathrm{P}<0.05,{ }^{* *} \mathrm{P}<0.01,{ }^{* * * *} \mathrm{P}<0.001$ vs. control group; ${ }^{\#} \mathrm{P}<0.05,{ }^{\# \#} \mathrm{P}<0.01,{ }^{\# \# \#} \mathrm{P}<0.001$ vs. VPA-treated group.

However, both VPA and NAC decreased the expression levels of 4-HNE and ROS (Fig. 2A and B). The above results demonstrated that NAC attenuated oxidative stress in the VPA-treated primary cultured neurons. Whether the decrease in oxidative stress downregulates the canonical Wnt signaling pathway in VPA-exposed primary cortical neurons remains unknown. Therefore, in further experiments, we investigated the changes occurring in the mRNA expression levels of $\beta$-catenin and GSK-3 $\beta$. The results from RT-qPCR revealed that GSK-3 $\beta$ expression was lower and $\beta$-catenin expression was higher in the VPA-treated group when compared with the controls; however treatment with both NAC and VPA did not alter the mRNA levels of GSK-3 $\beta$ and $\beta$-catenin in comparison to the VPA-treated group (Fig. 2C and D).

Effects of sulindac on VPA-exposed primary cultured neurons. Sulindac is a known pharmacological inhibitor of $\beta$-catenin, known for inhibiting $\beta$-catenin expression through proteasomedependent degradation and suppressing tumorigenesis in vivo by downregulating $\beta$-catenin signaling $(16,17)$. GSK-3 $\beta$ desta- 

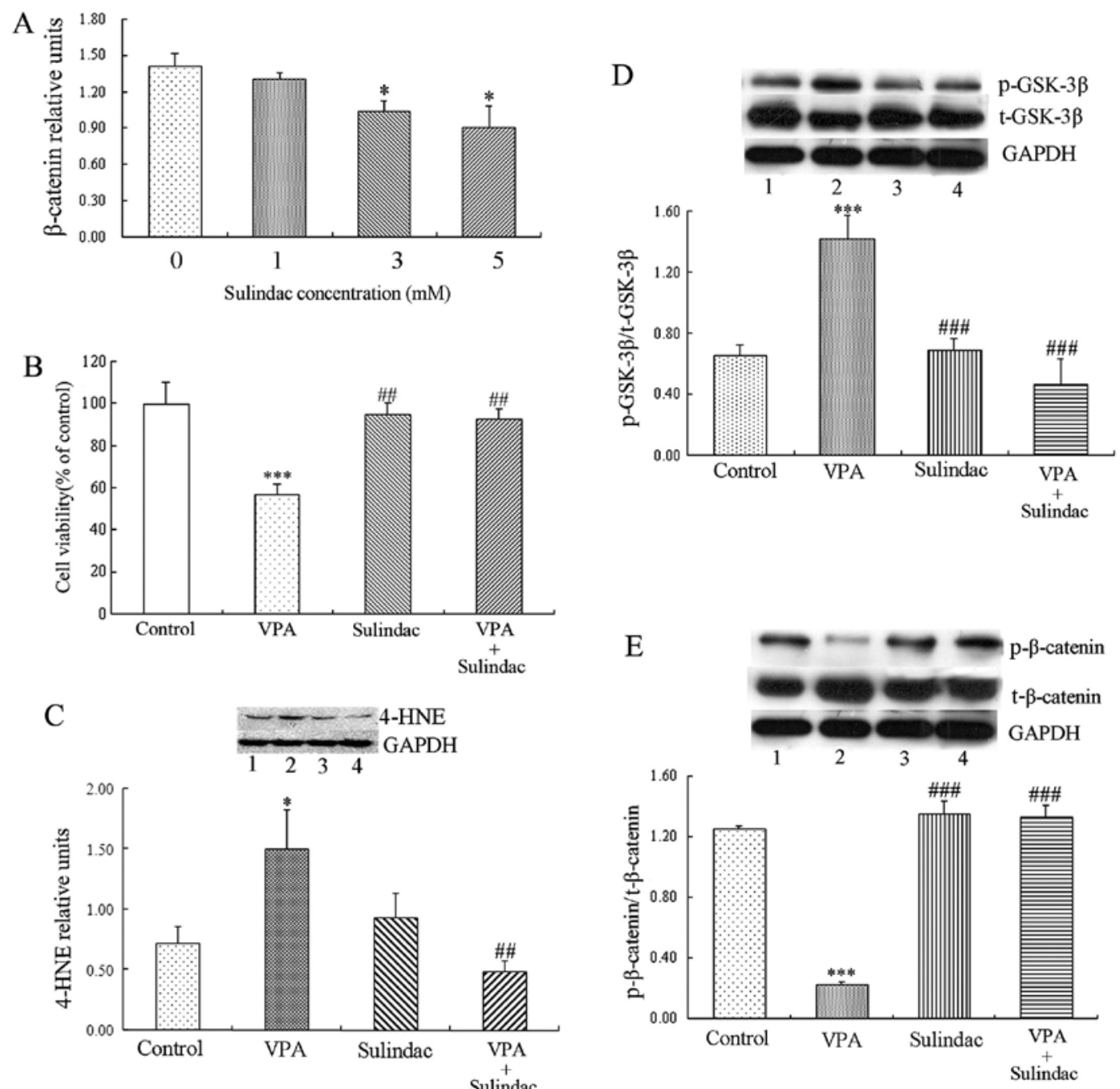

Figure 3. (A) RT-qPCR analysis of $\beta$-catenin epxression. (B) Representative cell viability detected by MTT assay. (C, D and E) Western blot analysis of 4-hydroxynonenal (4-HNE), total (t)-glycogen synthase kinase (GSK)-3 $\beta$, phosphorylated (p)-GSK-3 $\beta$, total (t)- $\beta$-catenin and phosphorylated (p)- $\beta$-catenin levels. ${ }^{\mathrm{P}} \mathrm{P}<0.05,{ }^{* * *} \mathrm{P}<0.001$ vs. control group; ${ }^{\# \mathrm{P}}<0.05,{ }^{\# \#} \mathrm{P}<0.01,{ }^{\# \# \#} \mathrm{P}<0.001$ vs. valproic acid (VPA)-treated group.

bilizes $\beta$-catenin by phosphorylating its inhibitory sites, such as Ser33, Ser37 and Thr41 (18). In this study, we thus detected the changes occurring in the Wnt/ $\beta$-catenin pathway, oxidative stress markers and cell viability in primary cortical neurons exposed to VPA following treatment with sulindac. Primary cultured neurons were exposed to VPA $(5 \mathrm{mmol} / \mathrm{l})$. After $24 \mathrm{~h}$, VPA was replaced with sulindac $(1,3$ and $5 \mathrm{mmol} / \mathrm{l})$ for $1 \mathrm{~h}$. The neurons were then harvested for RNA and protein extraction. The results from RT-qPCR revealed that treatment with sulindac decreased the mRNA levels of $\beta$-catenin (Fig. 3A). As shown in Fig. 3A, treatment with 3 and $5 \mathrm{mmol} / \mathrm{l}$ sulindac decreased the mRNA levels of $\beta$-catenin compared with the controls. Thus, we selected the dose of $3 \mathrm{mmol} / \mathrm{l}$ sulindac as the optimal concentration for further experiments. Whether treatment with $3 \mathrm{mmol} / \mathrm{l}$ sulindac inactivates the canonical Wnt pathway requires further verification. Therefore, we examined the phosphorylation levels of GSK-3 $\beta$ and $\beta$-catenin. Western blot analysis demonstrated that VPA activated the canonical Wnt pathway based on the increased phosphorylation levels of GSK-3 $\beta$, and the decreased phosphorylation levels of $\beta$-catenin in comparison to the controls (Fig. 3D and E). However, treatment with both sulindac and VPA inactivated the canonical Wnt pathway, as indicated by the decreased phosphorylation levels of GSK-3 $\beta$ and the increased phosphorylation levels of $\beta$-catenin compared with the VPA-treated group (Fig. 3D and E). We then detected the effects of sulindac on the viability of primary cultured cortical neurons. The results revealed that exposure to VPA decreased cell viability (Fig. 3B). However, co-incubation of the neurons with VPA and sulindac increased cell viability (Fig. 3B).

In order to investigate the effects of sulindac on oxidative stress, we determined the expression levels of the oxidative stress marker, 4-HNE. Western blot analysis revealed that the protein expression of 4-HNE was higher in the VPA-treated group than in the controls, whereas it was lower in the VPA plus sulindactreated group than in the VPA-treated group. However, the expression of 4-HNE was not altered in the VPA plus sulindactreated group compared with the control group (Fig. 3C).

Effects of sulindac on repetitive/stereotypic-like movements in Wistar rats prenatally exposed to VPA. The VPA-treated rats were hyperactive at the 0-5, 5-10 and 20-25 min intervals, demonstrating that they spent more time engaged in repetitive/stereotypic-like behaviors and had more repetitive/ stereotypic-like movements, with the results approaching 

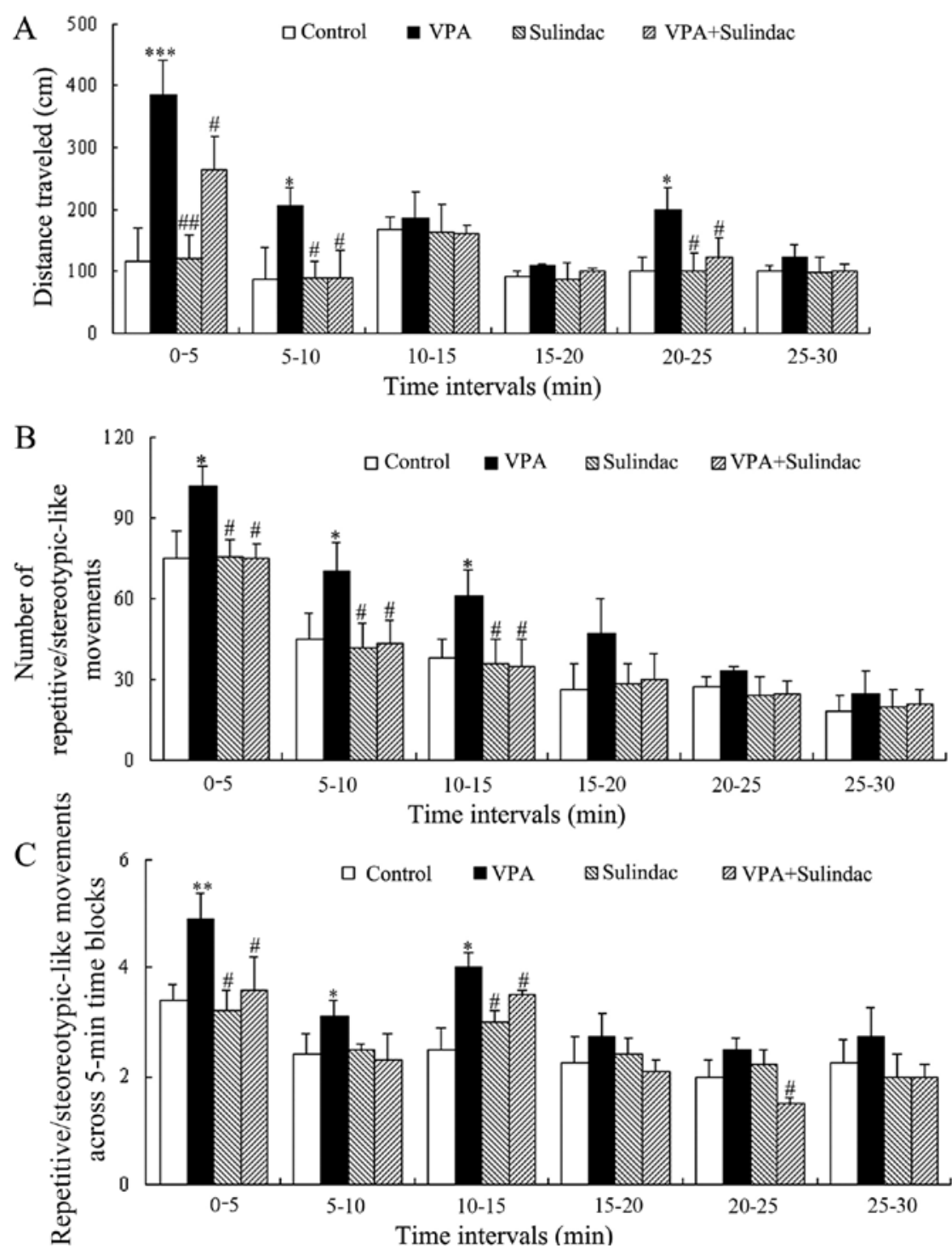

Figure 4. Distance travelled (A), the number of repetitive/stereotypic-like movements (B) and time spent in repetitive/stereotypic-like behavioral activities (C) measured across 5-min time blocks in 30 -min sessions. ${ }^{*} \mathrm{P}<0.05,{ }^{* *} \mathrm{P}<0.01,{ }^{* * *} \mathrm{P}<0.001$ vs. control rats; ${ }^{*} \mathrm{P}<0.05,{ }^{\# \#} \mathrm{P}<0.01$ vs. valproic acid (VPA)-exposed rats.

statistical significance at the 0-5, 5-10 and 10-15 min intervals in comparison to the controls (Fig. 4B and C). An increase in the distance traveled during the $0-5,5-10$ and 20-25 $\mathrm{min}$ intervals was also observed (Fig. 4A). Compared with the VPA-treated rats, the VPA plus sulindac-treated rats were hypoactive showing a decrease in distance traveled during the 0-5, 5-10 and 20-25 min intervals (Fig. 4A), a decrease in the number of repetitive/stereotypic-like behaviors during the $0-5$, 5-10 and 10-15 min intervals, as well as in the time engaged in repetitive/stereotypic-like movements during the $0-5,10-15$ and 20-25 min intervals (Fig. 4B and C). Additionally, selfgrooming and rearing behaviors were also scored during the open field locomotor activity task. The VPA-treated animals spent a significantly longer period of time in self-grooming activities compared to the controls (Fig. 5B). There was also a marked increase observed in the number of rearing episodes in the VPA-treated animals compared to the control animals (Fig. 5A). In comparison to the VPA-treated rats, the VPA plus sulindac-treated rats were hypoactive with a decreased number of self-grooming and rearing behavioral activities (Fig. 5A and B). There were no differences observed between the VPA plus sulindac-treated rats and the controls in the total activity across the 30-min testing session.

\section{Discussion}

The present study demonstrates the antioxidant properties of the NSAID, sulindac, based on a decrease in VPA-induced 4-HNE expression and ROS production following treatment with sulindac. The anti-inflammatory agent also protected rat cortical neurons against VPA-induced neurotoxicity, and ameliotared the repetitive/stereotypic behavioral abnormalities in Wistar rats prenatally exposed to VPA as a rat model of autism.

VPA, an anticonvulsant and mood-stabilizing drug, is widely used for the treatment of different types of seizures and myoclonic epilepsy (19). Several mechanisms have been suggested for VPA hepatotoxicity, and most of them are associated with oxidative stress. VPA has been shown to induce ROS production and lipid peroxidation formation (20), induce DNA damage (21) and decrease cell viability in hippocampal 


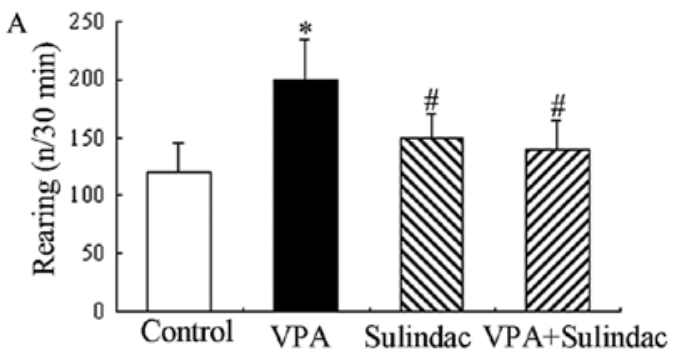

B

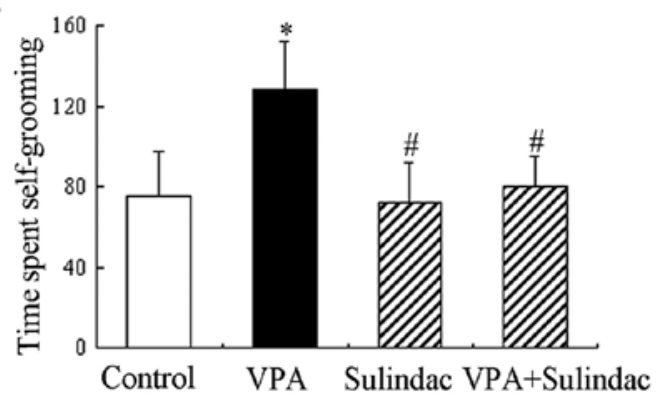

Figure 5. (A) The number of rearings, and (B) time spent in self-grooming during 30 -min sessions. ${ }^{*} \mathrm{P}<0.05$ vs. control rats; ${ }^{\#} \mathrm{P}<0.05$ vs. valproic acid (VPA)-exposed rats.

neurons (22). In agreement with previous studies, this study demonstrated that VPA increased ROS production and 4-HNE expression, thus inducing oxidative stress, and subsequently reducing neuronal viability. However, the co-incubation of the neurons with VPA and sulindac reduced the VPA-induced oxidative stress and increased neuronal viability. It is possible that the antioxidant and free radical scavenging properties of sulindac (23), are responsible for these neuroprotective effects. According to our previous results (4), the mechanisms through which sulindac exerts its antioxidant and free radical scavenging properties involve the upregulation of the $\beta$-catenin phosphorylation to suppress the activation of the canonical Wnt signaling pathway, leading to the downregulation of the downstream target genes, cyclin D1 and engrailed 2; these results are in accordance with those of previous studies demonstrating that sulindac is a specific inhibitor of the Wnt/ $\beta$-catenin pathway $(12,13)$.

Motor stereotypies are defined as patterned, repetitive, purposeless movements. These stigmatizing motor behaviors represent a manifestation of the third core criterion required for the diagnosis of autism, and are being viewed as potential early markers suggestive of autism (24), and hamper normal brain development, learning and social adaption. Moreover, motor stereotypies may be a tangible expression of the underlying neurobiology of this neurodevelopmental disorder. Furthermore, given the limited advances made towards unraveling the neurobiology of autism through the study of cardinal non-motor manifestations, such as language disorder and social impairments, a growing number of studies have turned their attention to motor impairments and stereotypies as a potential source of additional insight into the pathophysiology of autism $(15,25,26)$.

The present study demonstrated that VPA-treated rats showed a significant increase in the number of repetitive/stereotypiclike behavioral activities, which is largely in accordance with previous behavioral data $(27,28)$. This may be due to the reason that VPA-induced brain dysplasia, or increased glutamine transport leads to excessively excited brain neurons $(29,30)$. However, treatment of the animals with the NSAID, sulindac, improved the behavioral response, with the VPA plus sulindac-treated rats performing better than the VPA-treated animals, perhaps due to the reason that sulindac reduces the glutamate concentration or glutamine transport (31). Although sulindac improved performance in the open field test, it failed to completely suppress the VPA-induced motor deficits, as these rats showed more repetitive/stereotypic-like behavioral activities at certain time points compared to the control animals.

It has been shown that $N$-methyl D-aspartate NMDA receptor antagonists result into stereotypic movement disorder $(32,33)$, while sulindac increases the NR1 and NR2B NMDA receptor subunits in aged Fischer 344 rats (34). NMDA receptors are vital for brain function and these receptors are central to many of the activity-dependent changes occurring in synaptic strength and connectivity (35). It has been demonstrated that VPA reduces NMDA signaling and NMDA receptor-interacting protein expression in the rat brain (36). Sulindac prevents the age-related increase in the expression of the pro-inflammatory cytokine, interleukin-1 $\beta$ (1L-1 $\beta)$, in the hippocampus (34). It is possible that sulindac suppresses the inflammatory response in the brain, as well as scavenging ROS and protects the cortical neurons from VPA-induced neurotoxicity. Sulindac possibly reduces the loss of NMDA receptors and prevents the decrease in the number of cortical neurons, thereby averting VPA-induced stereotypic movement disorder.

The principal defense systems against oxygen free radicals are superoxide dismutase (SOD), glutathione (GSH), GSH peroxidases, GSH reductase, catalase and antioxidant nutrients. GSH and the related enzymes are present in the majority of cells. Imbalances in the expression of GSH and associated enzymes have been implicated in the pathogenesis of autism $(2,37)$. It has been demonstrated that VPA decreases GSH reductase activity with a subsequent decrease in GSH levels $(38,39)$, and increases glutathione peroxidase activity (40). The ratio of GSH/GSSG is a good measure of the oxidative stress of an organism. This study demonstrated that VPA enhanced 4-HNE and ROS levels, suggesting the generation of oxidative stress. However, treatment with the NSAID, sulindac, attenuated the increase in 4-HNE levels. It has been demonstrated that antioxidants prevent NMDA receptor depletion (41), suggesting that these NSAIDs also serve as antioxidants and prevent the depletion of NMDA receptors. This is another possible mechanism through which these NSAIDs improve performance in the open field test since it has been suggested that NMDA receptors are important during the acquisition of normal stereotypic movements (42).

In conclusion, the results from the present study led us to hypothesize that the canonical Wnt signaling pathway induces oxidative stress and therefore contributes to the susceptibility to autism. Sulindac, with antioxidant properties associated with the inhibition of the activition of the canonical Wnt pathway, protects primary cortical neurons, as well as rats against VPA-induced oxidative stress. Sulindac also improves stereotypic movement disorders that are adversely affected by VPA. The findings of the present study further support the argument that sulindac may play a beneficial role in neurodevelopmental disorders, such as autism. 


\section{Acknowledgements}

This study was financially supported by grants from the National Natural Science Foundation of China (nos. 81301174, 81260211 and 81271466) and the Doctoral Scientific Research Foundation of Xinxiang Medical University.

\section{References}

1. Ghanizadeh A and Derakhshan N: N-acetylcysteine for treatment of autism, a case report. J Res Med Sci 17: 985-987, 2012.

2. Al-Ayadhi LY and Elamin NE: Camel milk as a potential therapy as an antioxidant in autism spectrum disorder (ASD). Evid Based Complement Alternat Med 2013: 602834, 2013.

3. Wang Z,Xu L,Zhu X, etal: Demethylation of specific Wnt/ $\beta$-catenin pathway genes and its upregulation in rat brain induced by prenatal valproate exposure. Anat Rec 293: 1947-1953, 2010.

4. Zhang Y, Sun Y, Wang F, Wang Z, Peng Y and Li R: Downregulating the canonical Wnt/ $\beta$-catenin signaling pathway attenuates the susceptibility to autism-like phenotypes by decreasing oxidative stress. Neurochem Res 13: 1409-1419, 2012.

5. Hall AC, Lucas FR and Salinas PC: Axonal remodeling and synaptic differentiation in the cerebellum is regulated by WNT-7a signaling. Cell 100: 525-535, 2000.

6. Chenn A and Walsh CA: Regulation of cerebral cortical size by control of cell cycle exit in neural precursors. Science 297: 365-369, 2002.

7. Brault V, Moore R, Kutsch S, et al: Inactivation of the beta-catenin gene by Wnt1-Cre-mediated deletion results in dramatic brain malformation and failure of craniofacial development. Development 128: 1253-1264, 2001.

8. Martin PM, Yang X, Robin N, et al: A rare WNT1 missense variant overrepresented in ASD leads to increased Wnt signal pathway activation. Transl Psychiatry 3: e301, 2013.

9. Atkin TA, MacAskill AF, Brandon NJ and Kittler JT: Disrupted in Schizophrenia-1 regulates intracellular trafficking of mitochondria in neurons. Mol Psychiatry 16: 122-124, 121, 2011.

10. Bošković M, Grabnar I, Terzič T, Kores Plesničar B and Vovk T: Oxidative stress in schizophrenia patients treated with longacting haloperidol decanoate. Psychiatry Res 210: 761-768, 2013.

11. Crespi B, Stead P and Elliot M: Evolution in health and medicine Sackler colloquium: Comparative genomics of autism and schizophrenia. Proc Natl Acad Sci USA 107 (Suppl 1): 1736-1741, 2010

12. Lemjabbar-Alaoui H, Dasari V, Sidhu SS, et al: Wnt and Hedgehog are critical mediators of cigarette smoke-induced lung cancer. PLoS One 1: e93, 2006.

13. Huang Y, Chang X, Lee J, et al: Cigarette smoke induces promoter methylation of single-stranded DNA-binding protein 2 in human esophageal squamous cell carcinoma. Int J Cancer 128: 2261-2273, 2011.

14. Hansen MB, Nielsen SE and Berg K: Re-examination and further development of a precise and rapid dye method for measuring cell growth/cell kill. J Immunol Methods 119: 203-210, 1989.

15. Pearson BL, Pobbe RL, Defensor EB, et al: Motor and cognitive stereotypies in the BTBR $\mathrm{T}+\mathrm{tf} / \mathrm{J}$ mouse model of autism. Genes Brain Behav 10: 228-235, 2011.

16. Orner GA, Dashwood WM, Blum CA, Diaz GD, Li Q and Dashwood RH: Suppression of tumorigenesis in the Apc(min) mouse: down-regulation of beta-catenin signaling by a combination of tea plus sulindac. Carcinogenesis 24: 263-267, 2003.

17. Rice PL, Kelloff J, Sullivan H, et al: Sulindac metabolites induce caspase- and proteasome-dependent degradation of beta-catenin protein in human colon cancer cells. Mol Cancer Ther 2: 885-892, 2003.

18. Toledo EM, Colombres $M$ and Inestrosa NC: Wnt signaling in neuroprotection and stem cell differentiation. Prog Neurobiol 86 281-296, 2008.

19. Löscher W: Basic pharmacology of valproate: a review after 35 years of clinical use for the treatment of epilepsy. CNS Drugs 16: 669-694, 2002.

20. Jafarian I, Eskandari MR, Mashayekhi V, Ahadpour M and Hosseini MJ: Toxicity of valproic acid in isolated rat liver mitochondria. Toxicol Mech Methods 23: 617-623, 2013.

21. Khan S, Ahmad T, Parekh CV, Trivedi PP, Kushwaha S and Jena $\mathrm{G}$ : Investigation on sodium valproate induced germ cell damage, oxidative stress and genotoxicity in male Swiss mice. Reprod Toxicol 32: 385-394, 2011
22. Wang C, Luan Z, Yang Y, Wang Z, Cui Y and Gu G: Valproic acid induces apoptosis in differentiating hippocampal neurons by the release of tumor necrosis factor-alpha from activated astrocytes. Neurosci Lett 497: 122-127, 2011.

23. Santos F, Teixeira L, Lúcio M, et al: Interactions of sulindac and its metabolites with phospholipid membranes: an explanation for the peroxidation protective effect of the bioactive metabolite. Free Radic Res 42: 639-650, 2008.

24. Goldman S, O'Brien LM, Filipek PA, Rapin I and Herbert MR: Motor stereotypies and volumetric brain alterations in children with Autistic Disorder. Res Autism Spectr Disord 7: 82-92, 2013.

25. Lemonda BC, Holtzer R and Goldman S: Relationship between executive functions and motor stereotypies in children with Autistic Disorder. Res Autism Spectr Disord 6: 1099-1106, 2012.

26. Hattier MA, Matson JL, Macmillan K and Williams L: Stereotyped behaviours in children with autism spectrum disorders and atypical development as measured by the BPI-01. Dev Neurorehabil 16: 291-300, 2013.

27. Schneider T and Przewlocki R: Behavioral alterations in rats prenatally exposed to valproic acid: animal model of autism. Neuropsychopharmacology 30: 80-89, 2005.

28. Schneider T, Turczak J and Przewlocki R: Environmental enrichment reverses behavioral alterations in rats prenatally exposed to valproic acid: issues for a therapeutic approach in autism. Neuropsychopharmacology 31: 36-46, 2006.

29. Markram K, Rinaldi T, La Mendola D, Sandi C and Markram H: Abnormal fear conditioning and amygdala processing in an animal model of autism. Neuropsychopharmacology 33: 901-912, 2008.

30. Rinaldi T, Silberberg G and Markram H: Hyperconnectivity of local neocortical microcircuitry induced by prenatal exposure to valproic acid. Cereb Cortex 18: 763-770, 2008.

31. Bass SE, Sienkiewicz P, Macdonald CJ, et al: Novel dithiolethione-modified nonsteroidal anti-inflammatory drugs in human hepatoma HepG2 and colon LS180 cells. Clin Cancer Res 15: 1964-1972, 2009.

32. Pitsikas N, Zisopoulou S and Sakellaridis N: Nitric oxide donor molsidomine attenuates psychotomimetic effects of the NMDA receptor antagonist MK-801. J Neurosci Res 84: 299-305, 2006.

33. Pitsikas N, Zisopoulou S, Pappas I and Sakellaridis N: The selective 5-HT(6) receptor antagonist Ro 04-6790 attenuates psychotomimetic effects of the NMDA receptor antagonist MK-801. Behav Brain Res 188: 304-309, 2008.

34. Mesches MH, Gemma C, Veng LM, et al: Sulindac improves memory and increases NMDA receptor subunits in aged Fischer 344 rats. Neurobiol Aging 25: 315-324, 2004.

35. Bliss TV and Collingridge GL: A synaptic model of memory: long-term potentiation in the hippocampus. Nature 361: 31-39, 1993.

36. Basselin M, Chang L, Chen M, Bell JM and Rapoport SI: Chronic administration of valproic acid reduces brain NMDA signaling via arachidonic acid in unanesthetized rats. Neurochem Res 33: 2229-2240, 2008.

37. Kern JK, Haley BE, Geier DA, Sykes LK, King PG and Geier MR: Thimerosal exposure and the role of sulfation chemistry and thiol availability in autism. Int J Environ Res Public Health 10: 3771-3800, 2013.

38. Hsieh CL, Wang HE, Tsai WJ, Peng CC and Peng RY: Multiple point action mechanism of valproic acid-teratogenicity alleviated by folic acid, vitamin $\mathrm{C}$, and $\mathrm{N}$-acetylcysteine in chicken embryo model. Toxicology 291: 32-42, 2012.

39. Tunali S: The effects of vitamin B6 on lens antioxidant system in valproic acid-administered rats. Hum Exp Toxicol 33: 623-628, 2013.

40. Zhang Z, Qin X, Zhao X, et al: Valproic acid regulates antioxidant enzymes and prevents ischemia/reperfusion injury in the rat retina. Curr Eye Res 37: 429-437, 2012.

41. Patel AP, John MA, Handy RD and Robert SJ: Carbon monoxide exposure in rat heart: glutathione depletion is prevented by antioxidants. Biochem Biophys Res Commun 302: 392-396, 2003.

42. Deutsch SI, Pepe GJ, Burket JA, Winebarger EE, Herndon AL and Benson AD: D-cycloserine improves sociability and spontaneous stereotypic behaviors in 4-week old mice. Brain Res 1439: 96-107, 2012. 